\title{
RECONSTRUCTION OF THE ANTERIOR TALOFIBULAR LIGAMENT FOR THE TREATMENT OF THE UNSTABLE ANKLE
}

\author{
G. K. SEFTON, J. GEORGE, J. M. FITTON, H. McMULlEN \\ From St James's University Hospital, Leeds, and Victoria Hospital, Worksop
}

\begin{abstract}
Chronic instability of the ankle is frequently due to disruption of the fibres of the anterior talofibular ligament and the anterolateral capsule. This allows momentary subluxations at the ankle joint; the talus tilts into an abnormal varus position and rotates forwards and inwards about a vertical axis which passes through the medial malleolus. The use of a free tendon graft to reconstruct the anterior talofibular ligament restores the stability of the ankle without restricting movement at the subtalar level.
\end{abstract}

The detailed anatomy of the ligaments stabilising the lateral side of the ankle has been well documented. The main components are the anterior talofibular, the calcaneofibular and the posterior talofibular ligaments, which are reinforced by the capsular fibres. The recent literature gives conflicting opinions as to the site and extent of the lesion in chronic inversion injuries. Aufranc (1958) states that of all the ligamentous injuries those of the ankle, especially the fibular collateral ligaments, are the commonest and the least understood.

The anterior talofibular ligament is a distinct and sizeable structure extending from the anterior border of the fibula to the neck of the talus. Ala-Ketola et al. (1977) have shown by arthrography that it is the ligament most commonly injured. Work by Leonard (1949) and Anderson and LeCocq (1954) suggests that rupture of this element of the complex allows anterior displacement of the talus from the mortise of the joint in the coronal plane, displacement of the talus by medial rotation about its vertical axis, and talar tilting of 7 to 10 degrees because of the associated damage to the lateral capsule.

The calcaneofibular ligament extends from the tip of the fibula to the lateral surface of the calcaneus. The fibres are horizontal and relaxed when the ankle is inverted (Grond 1973) and therefore this ligament lacks major significance in the common recurrent inversion injury (Boström 1966). The posterior talofibular ligament is a wide strong band arising from the posterior surface of the fibula and running to the posterior aspect of the talus. Clinical disruption of this ligament is very rare (Boström 1966).

Although clinical and experimental work shows that injury to the anterior talofibular ligament is the main primary cause of persistent instability of the ankle, numerous operations have been evolved to create tight ligamentous structures between the lateral malleolus and the calcaneus or between the lateral malleolus and the fifth metatarsal. These procedures restrict inversion at the subtalar joint unnecessarily. It is more logical to reconstruct only the anterior talofibular ligament using a free tendon graft. This corrects the instability of the ankle without restricting inversion at the subtalar joint.

An operation has been devised which fulfils this requirement. It has been used for many years and provides excellent long-term stability without restriction of inversion.

\section{CLINICAL EXAMINATION}

The diagnosis of chronic instability of the ankle depends on clinical and radiological signs. Symptoms invariably span many years with recurrent inversion injuries, pain and swelling. The important feature on examination is abnormal movement of the talus in the ankle mortise. To demonstrate this, stress must be applied to cause inversion of the foot and to displace the talus forward in relation to the tibia and lateral malleolus. The anterior rotational displacement is then easily felt by palpation of the talus in front of the lateral malleolus. Boström (1966) referred to this as the "anterior drawer" sign. In comparison Gillespie and Boucher (1971) found evaluation of pure talar tilt was difficult and not as helpful.

Stress radiography is used to confirm the diagnosis. However, an abnormality of talar tilt does not always correlate with functional instability of the ankle (Freeman 1965) and varus tilting of up to 20 degrees may occur in otherwise normal ankles (Rubin and Witten 1960). As asymmetry is rarely found (Laurin and Mathieu 1975), the critical sign is a talar tilt substantially greater on the symptomatic side.

G. K. Sefton, F.R.C.S.Ed., Senior Orthopaedic Registrar, St James's University Hospital, Leeds LS9 7TF, England. J. George, M.D., Os Rzezzypospolitey 3/93, 61/397 Poznan, Poland.

J. M. Fitton, F. R.C.S., Consultant Orthopaedic Surgeon, St James's University Hospital, Leeds LS9 7TF, England.

H. McMullen, F.R.C.S., Consultant Orthopaedic Surgeon, Victoria Hospital, Worksop, Nottinghamshire, England.

Requests for reprints should be sent to Mr G. K. Sefton. 


\section{OPERATIVE TECHNIQUE}

The operation is done under general anaesthesia using an exsanguinating tourniquet. Material for the graft should preferably be derived from the plantaris tendon of the same leg using a Brand plantaris tendon stripper. If the plantaris muscle and tendon are absent, the tendons of the long extensors of the toes or the peroneus brevis are suitable alternatives

The anterolateral aspect of the ankle is exposed by an oblique incision passing forwards and slightly downwards from the posterior aspect of the fibula about an inch above the malleolus. The thin sheet of scar tissue which replaces the damaged capsule is easily stripped away to expose the lateral aspect of the neck and body of the talus and the lateral malleolus. It may be necessary to divide some extensor retinacular fibres to gain sufficient exposure to the lateral side of the neck of the talus.

Using Paton's burrs of appropriate diameter four drill holes are made as shown in Figure 1. The first hole passes horizontally through the fibula from its superficial surface to its anterior margin a little below the level of the horizontal plane of the ankle joint. The second hole goes downwards through the outer side of the neck of the talus, which presents a firm and convenient ridge that is easily perforated. The third hole runs horizontally backwards through the body of the talus in a plane that is deep to the lowest part of the fibular articular surface on its lateral aspect. It is, of course, difficult to construct these tunnels and still leave a good bony cover. Care must also be taken to avoid damaging the calcaneal surface of the talus. The fourth hole passes upwards through the lateral malleolus from its lowest point on to the surface, a little below the entry point for the first hole.

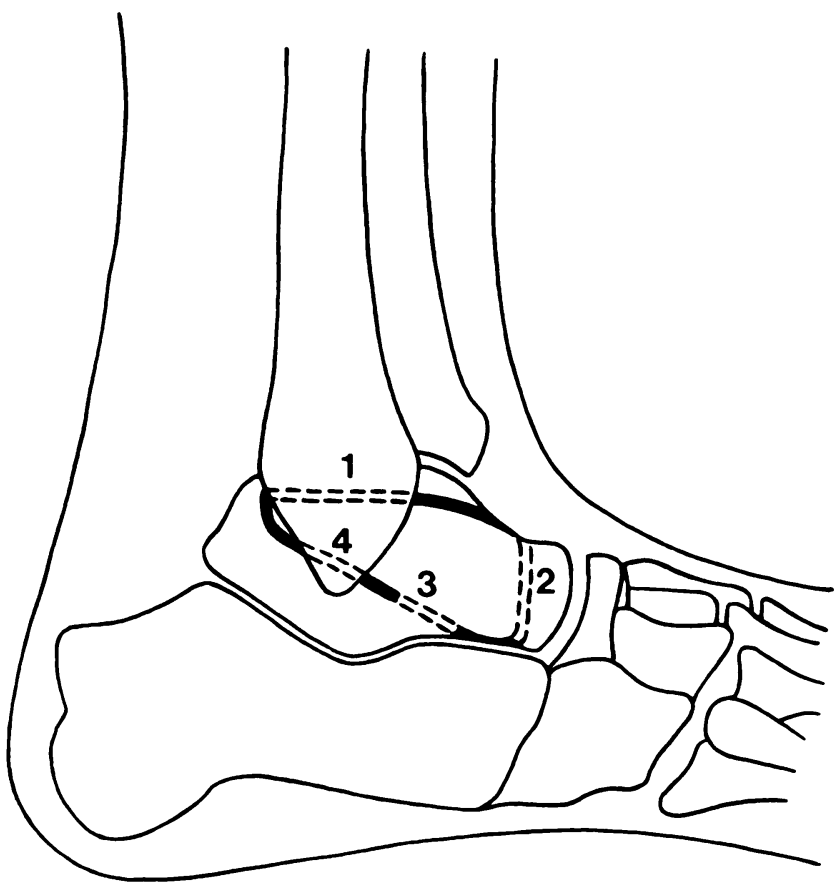

Fig. 1

Diagram to show the four drill holes through which the tendon graft is inserted.

The plantaris tendon is usually long enough to be passed in double thickness through these four holes. It is pulled tight, and the four ends of the graft are knotted together on the surface of the lateral malleolus and fixed by one or two anchoring silk sutures: it is then easily demonstrated that normal stability of the ankle joint has been restored. The foot and ankle are then immobilised at a right angle in a below-knee plaster. Weight-bearing through the plaster may be resumed when the wound has healed, and the plaster may be removed at six weeks to allow graduated weight-bearing activities.

\section{RESULTS}

During the last twelve years thirty-four ankles in thirty-four patients have been stabilised using this procedure but it has only been possible to examine eighteen. Six other patients reported that they were satisfied but did not attend for examination and so were not included in the review. Ten were lost to follow-up. The age at the time of operation had varied from ten to

Table I. Grading of symptoms in instability of the lateral ligament of the ankle
Grade $l$ Full activity, including strenuous sport. No pain, swelling or giving way.
Grade 2 Occasional aching only after strenuous exercise. No giving way or feeling of apprehension.
Grade 3 No giving way but some remaining apprehension. especially on rough ground.
Grade 4 Recurrent instability and giving way in normal activities. with episodes of pain and swelling

fifty-eight years (average twenty-nine years). All had complained of recurrent lateral instability with associated pain and swelling. The duration of symptoms before operation had ranged from six months to fifteen years with a mean of five years. The follow-up examinations were made after a period varying from six months to twelve years (average five years). All patients had been able to resume their normal occupations and those who wished were able to participate in their favourite sports.

The grading suggested by Good, Jones and Livingstone (1975) was used to evaluate the symptoms (Table I). The results were all satisfactory, sixteen being in Grade 1 and two in Grade 2. Clinical examination revealed that there was no restriction of ankle movement. Particular attention was directed to the assessment of subtalar movement. In sixteen cases inversion was normal, in the other two it was limited by 5 degrees. All the wounds healed uneventfully.

\section{DISCUSSION}

Operative repair of the lateral ligament of the ankle has been described by many authors (Evans 1953; WatsonJones 1955; Lee 1957; Good et al. 1975) but their procedures involve some form of lateral tenodesis by which the peroneus brevis tendon is fixed to the lower end of the fibula. They act by restricting inversion of the foot in relation to the leg and can only be effective if they limit normal movement at the subtaloid level. All of the patients operated on by Evans (1953) had limited inversion, as did those treated by Elmslie (1934).

A different approach to the problem, as first suggested by Burrows (1955), is to reconstruct the anterior talofibular ligament using a free tendon graft. This restores the normal anatomy and allows full movement of the ankle joint and the subtalar joint. This 
may prove absolutely essential for a young person wishing to resume a career in a professional sport. Indeed, one of our patients subsequently suffered a compound fracture-dislocation of his talus while playing rugby football. At exploration the reconstructed anterior ligament was intact.
The review demonstrated a prolonged period of dysfunction after operation before the patients felt their ankles were stable. This reflects Freeman's hypothesis that derangement of the proprioceptive protective mechanism is a factor in ankle instability, and that it takes some time for proprioceptive adaptation to occur.

This operation was devised by Mr J. M. Fitton and Mr H. McMullen and has been used at both hospitals for a number of years. The information presented in this paper is derived from a study of their patients.

\section{REFERENCES}

Ala-Ketola, L., Puranen, J., Koivisto, E., and Puuperä, M. (1977) Arthrography in the diagnosis of ligament injuries and classification of ankle injuries. Radiology, 125, 63-68.

Anderson, K. J., and LeCocq, J. F. (1954) Operative treatment of injury to the fibular collateral ligament of the ankle. Journal of Bone and Joint Surgery, 36-A, 825-832.

Aufranc, O. E. (1958) Ankle injuries, ligamentous injuries. In Fractures and Other Injuries, pp. 582-585. Edited by E. F. Cave. Chicago: The Year Book Publishers Inc.

Boström, L. (1966) Sprained ankles. VI. Surgical treatment of “chronic" ligament ruptures. Acta Chirurgica Scandinavica, 132, 551-565.

Burrows, H. J. (1955) Elmslie's operation for overlooked rupture of the lateral ligament of the ankle. Journal of Bone and Joint Surgery, 37-B, 169.

Elmslie, R. C. (1934) Recurrent subluxation of the ankle-joint. Annals of Surgery, 100, 364-367.

Evans, D. L. (1953) Recurrent instability of the ankle-a method of surgical treatment. Proceedings of the Royal Society of Medicine. 46, 343-344.

Freeman, M. A. R. (1965) Instability of the foot after injuries to the lateral ligament of the ankle. Journal of Bone and Joint Surgery. 47-B, 669-677.

Gillespie, H. S., and Boucher, P. (1971) Watson-Jones repair of lateral instability of the ankle. Journal of Bone and Joint Surgery, 53-A, 920-924.

Good, C. J., Jones, M. A., and Livingstone, B. N. (1975) Reconstruction of the lateral ligament of the ankle. Injury. 7, 63-65.

Grond, J. T. H. (1973) The surgical treatment of injuries of the fibular collateral ligaments of the ankle. Archivum Chirurgicum Neerlandicum, 25, $131-136$.

Laurin, C., and Mathieu, J. (1975) Sagittal mobility of the normal ankle. Clinical Orthopaedics and Related Research, $108,99-104$.

Lee, H. G. (1957) Surgical repair in recurrent dislocation of the ankle joint. Journal of Bone and Joint Surgery, 39-A, 828-834.

Leonard, M. H. (1949) Injuries of the lateral ligaments of the ankle. Journal of Bone and Joint Surgery, 31-A, 373-377.

Rubin, G., and Witten, M. (1960) The talar-tilt angle and the fibular collateral ligaments. Journal of Bone and Joint Surgery. 42-A, $311-326$.

Watson-Jones, Sir R. (1955) Fractures and Joint Injuries. Fourth edition. Edinburgh and London: E. and S. Livingstone Ltd. 